\section{Sensitivity, specificity, and predictive value of cardiac symptoms assessed by emergency medical services providers in the diagnosis of acute myocardial infarction: a multi-center observational study}

\author{
Jeong Ho Park ${ }^{1,2}$, Sung Woo Moon ${ }^{1,3}$, Tae Yun Kim, ${ }^{1,4}$, Young Sun Ro ${ }^{1}$, \\ Won Chul Cha ${ }^{1,5}$, Yu Jin Kim ${ }^{1,6}$, Sang Do Shin ${ }^{1,2}$ \\ 'Laboratory of Emergency Medical Services, Seoul National University Hospital Biomedical Research
Institute, Seoul, Korea \\ ${ }^{2}$ Department of Emergency Medicine, Seoul National University Hospital, Seoul National University College \\ of Medicine, Seoul, Korea \\ ${ }^{3}$ Department of Emergency Medicine, Korea University Ansan Hospital, Korea University College of \\ Medicine, Ansan, Korea \\ ${ }^{4}$ Department of Emergency Medicine, Gyeongsang National University Hospital, Gyeongsang National \\ University College of Medicine, Jinju, Korea \\ ${ }^{5}$ Department of Emergency Medicine, Samsung Medical Center, Sungkyunkwan University School of \\ Medicine, Seoul, Korea \\ ${ }^{6}$ Department of Emergency Medicine, Seoul National University Bundang Hospital, Seoul National \\ University College of Medicine, Seongnam, Korea
}

Objective For patients with acute myocardial infarction (AMI), symptoms assessed by emergency medical services (EMS) providers have a critical role in prehospital treatment decisions. The purpose of this study was to evaluate the diagnostic accuracy of EMS provider-assessed cardiac symptoms of AMI.

Methods Patients transported by EMS to 4 study hospitals from 2008 to 2012 were included. Using EMS and administrative emergency department databases, patients were stratified according to the presence of EMS-assessed cardiac symptoms and emergency department diagnosis of AMI. Cardiac symptoms were defined as chest pain, dyspnea, palpitations, and syncope. Disproportionate stratified sampling was used, and medical records of sampled patients were reviewed to identify an actual diagnosis of AMI. Using inverse probability weighting, verification bias-corrected diagnostic performance was estimated.

Results Overall, 92,353 patients were enrolled in the study. Of these, 13,971 (15.1\%) complained of cardiac symptoms to EMS providers. A total of 775 patients were sampled for hospital record review. The sensitivity, specificity, positive predictive value, and negative predictive value of EMS provider-assessed cardiac symptoms for the final diagnosis of AMI was 73.3\% (95\% confidence interval [Cl], 70.8 to 75.7$), 85.3 \%(95 \% \mathrm{Cl}, 85.3$ to 85.4$), 3.9 \%(95 \% \mathrm{Cl}, 3.6$ to 4.2$)$, and $99.7 \%$ (95\% Cl, 99.7 to 99.8), respectively.

Conclusion We found that EMS provider-assessed cardiac symptoms had moderate sensitivity and high specificity for diagnosis of AMI. EMS policymakers can use these data to evaluate the pertinence of specific prehospital treatment of AMI.

Keywords Myocardial infarction; Emergency medical services; Symptom assessment; Sensitivity and specificity; Predictive value of tests
elSSN: 2383-4625

Received: 27 July 2017

Revised: 12 September 2017

Accepted: 26 September 2017

Correspondence to: Sung Woo Moon Department of Emergency Medicine, Korea University Ansan Hospital, 123 Jeokgeum-ro, Danwon-gu, Ansan 15355, Korea

E-mail: sungwoo.moon89@gmail.com ORCID

http://orcid.org/0000-0001-9950-3449

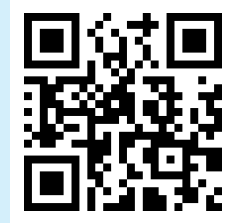

How to cite this article:

Park JH, Moon SW, Kim TY, Ro YS, Cha WC, Kim YJ, Shin SD. Sensitivity, specificity, and predictive value of cardiac symptoms assessed by emergency medical services providers in the diagnosis of acute myocardial infarction: a multi-center observational study. Clin Exp Emerg Med 2018;5(4):264-271.

This is an Open Access article distributed under the terms of the Creative Commons Attribution Non-Commercial License (http:// creativecommons.org/licenses/by-nc/4.0/). 


Capsule
Wumat is already known
In contrast to many studies about the diagnostic accuracy of hospital physician-assessed cardiac symptoms for acute
myocardial infarction (AMI), the diagnostic accuracy of emergency medical services (EMS) provider-assessed cardiac
symptoms for AMI has not been evaluated in previous studies.
What is new in the current study
The sensitivity of EMS provider-assessed cardiac symptom for the final diagnosis of AMI was $73.3 \%$ (95\% confidence
interval [CI], 70.8 to 75.7), specificity was $85.3 \%(95 \% \mathrm{Cl}, 85.3$ to 85.4$)$, positive predictive value was 3.9\% (95\% Cl, 3.6
to 4.2), and negative predictive value was $99.7 \%(95 \% \mathrm{Cl}, 99.7$ to 99.8$)$. We found that EMS provider-assessed cardiac
symptoms had moderate sensitivity and high specificity for diagnosis of AMI.

\section{INTRODUCTION}

The mortality rate of acute myocardial infarction (AMI) has declined substantially during the past 30 years. ${ }^{1,2}$ However, AMI is still a leading cause of death in many countries, ${ }^{3}$ and the importance of timely treatment is well established. ${ }^{4}$ Many AMI patients do not receive timely and proper treatment, and prehospital delay is among the main causes of delayed treatment..$^{5-7}$ In prehospital areas, various methods, including assessment via 12-lead electrocardiograms (ECGs), activation of the cardiac catheter laboratory, and fibrinolysis to shorten the time to reperfusion, are used to manage AMI patients. ${ }^{8-13}$

Although prehospital 12-lead ECG is the most studied tool and is a class I recommendation for the prehospital management of $\mathrm{AMI}_{1}^{8,14}$ whether a prehospital 12-lead ECG reading is taken or not is based on the symptoms of the patient and is yet to be determined. Therefore, emergency medical services (EMS) providerassessed symptoms have a critical role in evaluating AMI patients in prehospital settings. When AMI is accurately assessed in the prehospital phase, it is possible to provide optimal prehospital management and rapid transport, select a proper receiving hospital, and ultimately reduce first medical contact to device time in AMl patients. ${ }^{14}$

In contrast to many studies about the diagnostic accuracy of hospital physician-assessed cardiac symptoms for $\mathrm{AMI}_{1}{ }^{15}$ the diagnostic accuracy of EMS provider-assessed cardiac symptoms for AMI has not been evaluated in previous studies. We hypothesized that the diagnostic accuracy of EMS provider-assessed cardiac symptoms for AMI would be acceptable and associated with proper management and reduced process times in prehospital and hospital settings. This study aimed to describe the diagnostic accuracy of EMS provider-assessed cardiac symptoms for AMI and to identify the difference in prehospital and hospital processes according to the presence of EMS provider-assessed cardiac symptoms.

\section{METHODS}

\section{Study setting}

Korea established a single-tiered and fire-based public EMS system in 1995. The emergency hotline number in Korea is 119, and emergency medical technicians (EMTs) are dispatched during emergency calls. Detailed information on the education and training of Korean EMTs has been described previously. ${ }^{16}$ EMTs can provide care comparable to that of intermediate EMT level in the US, including intravenous fluid infusion, endotracheal intubation or laryngeal mask airway insertion, and administration of certain medications, including nitroglycerin, under online medical direction. ${ }^{17}$ An ambulance runsheet is filled out by EMTs for every ambulance dispatch, and all information is electronically recorded in the servers of the headquarters of the 16 provinces. Since a pilot trial of prehospital 12-lead ECG reading by the National Emergency Management Agency in 2009, EMS providers have acquired prehospital 12-lead ECG readings in some regions in Korea. However, it is not yet a widely accepted method for AMI management during emergencies.

\section{Study design and data collection}

This study was a retrospective observational study using an EMS database, administrative databases of participating emergency departments (EDs), and hospital medical records. The records of 4 large tertiary academic EDs located in urban areas with 40,000 to 80,000 annual patients were reviewed. We acquired the EMS database from the National Emergency Management Agency for this study. Using the EMS database from January 1, 2008 to December 31, 2012, we linked the participating hospitals' administrative data to the EMS database and assessed information on age, sex, visiting date, and visiting time. The hospital medical records of the sampled patients were reviewed by trained researchers. Available ED records, hospital admission records, nursing charts, and coronary angiography reports were reviewed. 


\section{Study population}

We included patients who visited any of the 4 participating hospitals' EDs by EMS from January 1, 2008 to December 31, 2012. Patients whose EMS data were not linked to the administrative data were excluded. Using the disproportionate stratified sampling method, we planned to enroll 800 patients for our analysis.

\section{Sampling and weighting}

Because patients who had EMS-assessed cardiac symptoms and patients whose final diagnosis was AMI were rare in the EMS database, a disproportionate stratified sample design was used to gain an adequate number of those patients. EMS-assessed cardiac symptoms and a discharge diagnosis of AMI in the administrative database were used for stratification. To gain an evenly distributed sample, the hospital and year of visit were also used for stratification. We planned to sample 10 patients in each stratum for a total of 800 patients. The sampled data were weighted to the probability of selection.

\section{Variables and measurement}

Among the 30 predefined categories of symptoms in the EMS database, we defined EMS-assessed cardiac symptoms as chest pain, dyspnea, palpitation, and syncope. Meanwhile, an ED discharge diagnosis of AMI was defined as an International Classification of Diseases 10th revision (ICD-10) code of I21.0 to I21.9 in the administrative ED database.

From the EMS database, we collected data on the patient's age, sex, symptom to call time, response time, scene time, transport time, hospital arriving time, presenting symptoms, prehospital documented shock (at least 1 event of systolic blood pressure less than $90 \mathrm{mmHg}$ as measured by the EMS), and prehospital management, including oxygen therapy, ECG monitoring, intravenous fluid infusion, nitroglycerin administration, and cardiopulmonary resuscitation (CPR).

From the participating hospital's administrative database, we collected data regarding patients' age, sex, hospital arriving time, ICD-10 ED diagnosis, and ED disposition status.

By reviewing hospital medical records, we collected data on the final diagnosis of AMI or not. For AMI patients, we also collected data on the presence of ST segment elevation, cardiogenic shock before reperfusion therapy, performance of CPR before reperfusion therapy, and the type of reperfusion therapy (thrombolysis, percutaneous coronary intervention, or coronary artery bypass grafting). Data on door-to-needle or balloon times were also collected in patients with ST elevation myocardial infarction (STE$\mathrm{MI})$.

\section{Statistical analysis}

Descriptive statistics were used to assess the similarity between the study population and the study sample. The characteristics of patients whose final diagnosis was AMI and STEMI were compared according to EMS provider-assessed cardiac symptoms. Values in the study sample were weighted via the reciprocal of the probability of selection in all analyses. Because we planned to compare weighted values according to EMS provider-assessed cardiac symptoms, design-based statistical tests, including Student's t-test, Wilcoxon rank-sum test, chi-square test, and Fisher exact test, were used as appropriate using a survey package for $\mathrm{R}$ software. The sensitivity, specificity, positive predictive value (PPV), and negative predictive value (NPV) with 95\% confidence interval (Cl) of each of the EMS-assessed cardiac symptoms as well as all of the EMS-assessed cardiac symptoms combined were calculated for the final diagnosis of AMI. Verification bias-adjusted estimates were calculated by using stratified sampling to weigh re-

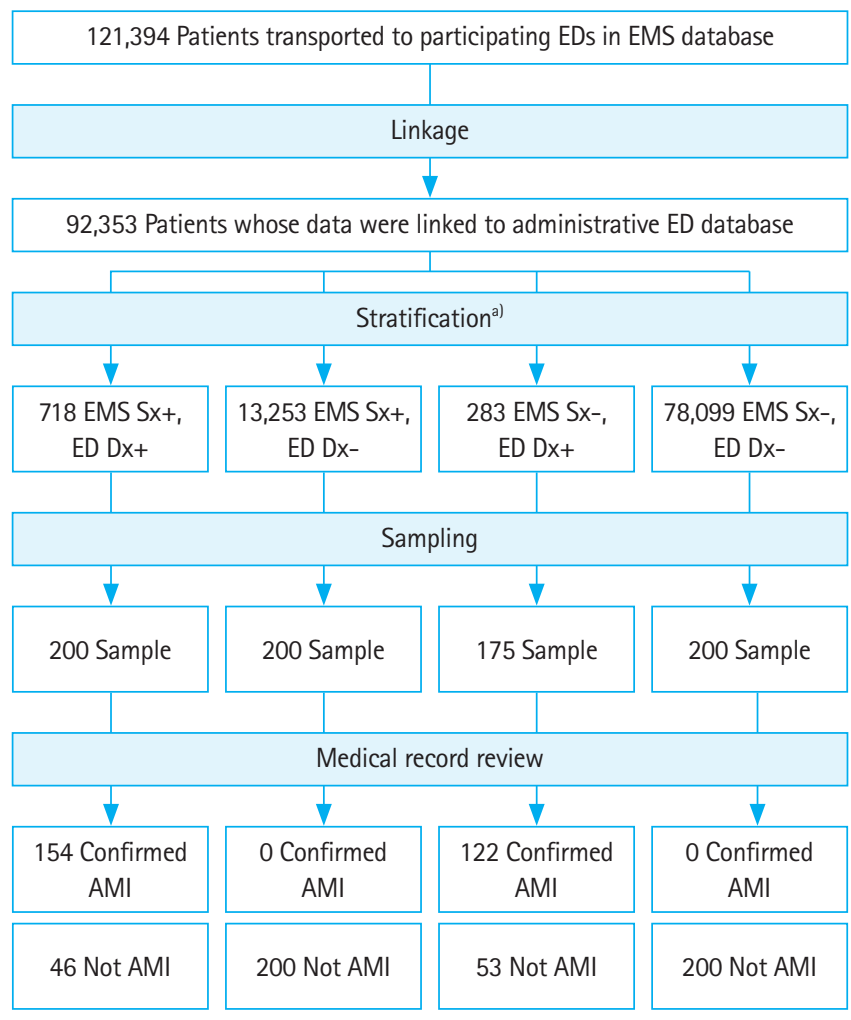

Fig. 1. Schematic of data analyses. ED, emergency department; EMS emergency medical services; EMS $\mathrm{Sx}+$, patients who had EMS providerassessed cardiac symptoms; ED Dx+, patients diagnosed with AMI in the administrative ED database; ED Dx-, patients who did not have a diagnosis of $\mathrm{AMI}$ in the administrative ED database; EMS Sx-, patients who did not have EMS provider-assessed cardiac symptoms; AMI, acute myocardial infarction. ${ }^{\text {a) }}$ Hospital and year of visit was also used in stratification. 
sults from patients in the EMS database using CompareTests for R software. ${ }^{18-20}$

\section{Ethics statement}

The study protocol was approved by the Institutional Review Board of the Seoul National University Hospital (1509-120-705). Informed consent was waived by the Institutional Review Board.

\section{RESULTS}

\section{Characteristics of the study participants}

From January 2008 to December 2012, 121,394 patients who visited the participating hospitals via EMS were identified from the EMS database. Among them, data of 92,353 (76.1\%) patients were linked to a participating hospital's administrative ED database. Although stratified sampling of 800 patients was planned, only 775 patients were sampled because the total number of patients in some strata did not exceed the planned number. The medical records of the sampled 775 patients were reviewed. Among them, 276 patients had a final diagnosis of AMI. Notably, no confirmed AMI was observed in patients who did not have a diagnosis of AMI in the administrative ED database (Fig. 1).

Table 1 shows the baseline characteristics of the study population and sample. Among all patients, $15.1 \%$ had any of the EMS provider-assessed cardiac symptoms, and $4.8 \%$ had chest pain. The mean age was 52.2 years in the total population, 58.5 years in the unweighted sample, and 49.2 years in the weighted sample. The proportion of men in the total population, unweighted sample, and weighted sample was 52.5\%, 59.6\%, and 51.5\%, respectively. Although baseline characteristics were different between all patients and sampled patients, the weighted values were similar between them.

Table 1. Prehospital and ED characteristics of the study population and sample

\begin{tabular}{|c|c|c|c|}
\hline Variable & $\begin{array}{c}\text { Total } \\
n=92,353\end{array}$ & $\begin{array}{l}\text { Sample (unweighted) } \\
\qquad n=775\end{array}$ & $\begin{array}{c}\text { Sample }\left(\text { weighted }^{\text {a) }} \text { ) }\right. \\
\qquad n=92,353\end{array}$ \\
\hline Age (yr) & $52.2 \pm 22.3$ & $58.5 \pm 19.4$ & $49.2 \pm 22.5$ \\
\hline Sex, male & $48,526(52.5)$ & $462(59.6)$ & $47,577(51.5)$ \\
\hline \multicolumn{4}{|c|}{ EMS provider-assessed cardiac symptoms } \\
\hline Chest pain & $4,413(4.8)$ & $200(25.8)$ & $3,470(3.8)$ \\
\hline Dyspnea & $7,764(8.4)$ & $185(23.9)$ & $8,494(9.2)$ \\
\hline Palpitations & $461(0.5)$ & $9(1.2)$ & $391(0.4)$ \\
\hline Syncope & $2,175(2.4)$ & $45(5.8)$ & $2,443(2.6)$ \\
\hline Any cardiac symptom & $13,971(15.1)$ & $375(51.6)$ & $13,971(15.1)$ \\
\hline Prehospital documented shock ${ }^{\mathrm{b})}$ & $5,319(5.8)$ & $76(9.8)$ & $8,082(8.8)$ \\
\hline \multicolumn{4}{|l|}{ Prehospital time interval (min) } \\
\hline Response time & $7.0(5.0-9.0)$ & $7.0(5.0-9.0)$ & $7.0(5.0-9.0)$ \\
\hline Scene time & $7.0(4.0-10.0)$ & $6(4-10)$ & $7.0(4.0-10.0)$ \\
\hline Transport time & $11.0(7.0-20.0)$ & $11.0(7.0-19.0)$ & $10.0(7.0-18.0)$ \\
\hline Total prehospital time & $28.0(21.0-38.0)$ & $26.0(20.0-36.0)$ & $26.0(20.0-36.0)$ \\
\hline \multicolumn{4}{|l|}{ Prehospital management } \\
\hline Oxygen therapy & $22,855(24.7)$ & $380(49.0)$ & $25,800(27.9)$ \\
\hline ECG monitoring & $16,016(17.3)$ & $266(34.3)$ & $13,262(14.4)$ \\
\hline IV infusion & $1,247(1.4)$ & $14(1.8)$ & $706(0.8)$ \\
\hline Nitroglycerin & $295(0.3)$ & $16(2.1)$ & $216(0.2)$ \\
\hline Cardiopulmonary resuscitation & $1,361(1.5)$ & $28(3.6)$ & $817(0.9)$ \\
\hline ED diagnosis of AMI & $1,001(1.1)$ & $375(48.4)$ & $1,001(1.1)$ \\
\hline \multicolumn{4}{|l|}{ ED disposition } \\
\hline Discharged & $58,111(62.9)$ & $235(30.3)$ & $55,852(60.5)$ \\
\hline Admitted & 25,777 (27.9) & $62.6(18)$ & 27,109 (29.4) \\
\hline Transferred & 3,504 (3.8) & $28(3.6)$ & $5,825(6.3)$ \\
\hline ED death & $1,811(2.0)$ & $18(2.3)$ & $1,396(1.5)$ \\
\hline Others & $1,693(1.8)$ & $9(1.2)$ & $2,172(2.4)$ \\
\hline
\end{tabular}

Values are presented as mean \pm standard deviation, number (\%), or median (interquartile range).

ED, emergency department; EMS, emergency medical services; ECG, electrocardiogram; IV, intravenous; AMI, acute myocardial infarction.

${ }^{a}$ Values are weighted in sample. ${ }^{b}$ Patients whose systolic blood pressure was less than $90 \mathrm{mmHg}$ in the EMS database at least once. 
Table 2. Diagnostic performance of EMS-assessed cardiac symptoms for acute myocardial infarction

\begin{tabular}{lcccc}
\hline EMS-assessed symptom & Sensitivity & Specificity & PPV & NPV \\
\hline Chest pain & $65.1(62.7-67.4)$ & $95.9(95.9-95.9)$ & $11.5(10.9-12.3)$ & $99.7(99.7-99.7)$ \\
Dyspnea & $12.9(11.3-14.6)$ & $91.6(91.6-91.6)$ & $1.2(1.1-1.4)$ & $99.2(99.2-99.3)$ \\
Palpitations & $0.3(0.3-0.3)$ & $99.9(99.9-99.9)$ & $1.9(1.9-1.9)$ & $99.2(99.2-99.3)$ \\
Syncope & $1.0(0.7-1.6)$ & $97.9(97.9-97.9)$ & $0.4(0.2-0.6)$ & $99.2(99.2-99.3)$ \\
Any cardiac symptom & $73.3(70.8-75.7)$ & $85.3(85.3-85.4)$ & $3.9(3.6-4.2)$ & $99.7(99.7-99.8)$ \\
\hline
\end{tabular}

Values are presented as \% (95\% confidence interval).

EMS, emergency medical services; PPV, positive predictive value; NPV, negative predictive value.

Table 3. Prehospital, ED, and hospital characteristics of confirmed AMI patients according to EMS provider-assessed cardiac symptom in the study sample $^{\mathrm{a})}$

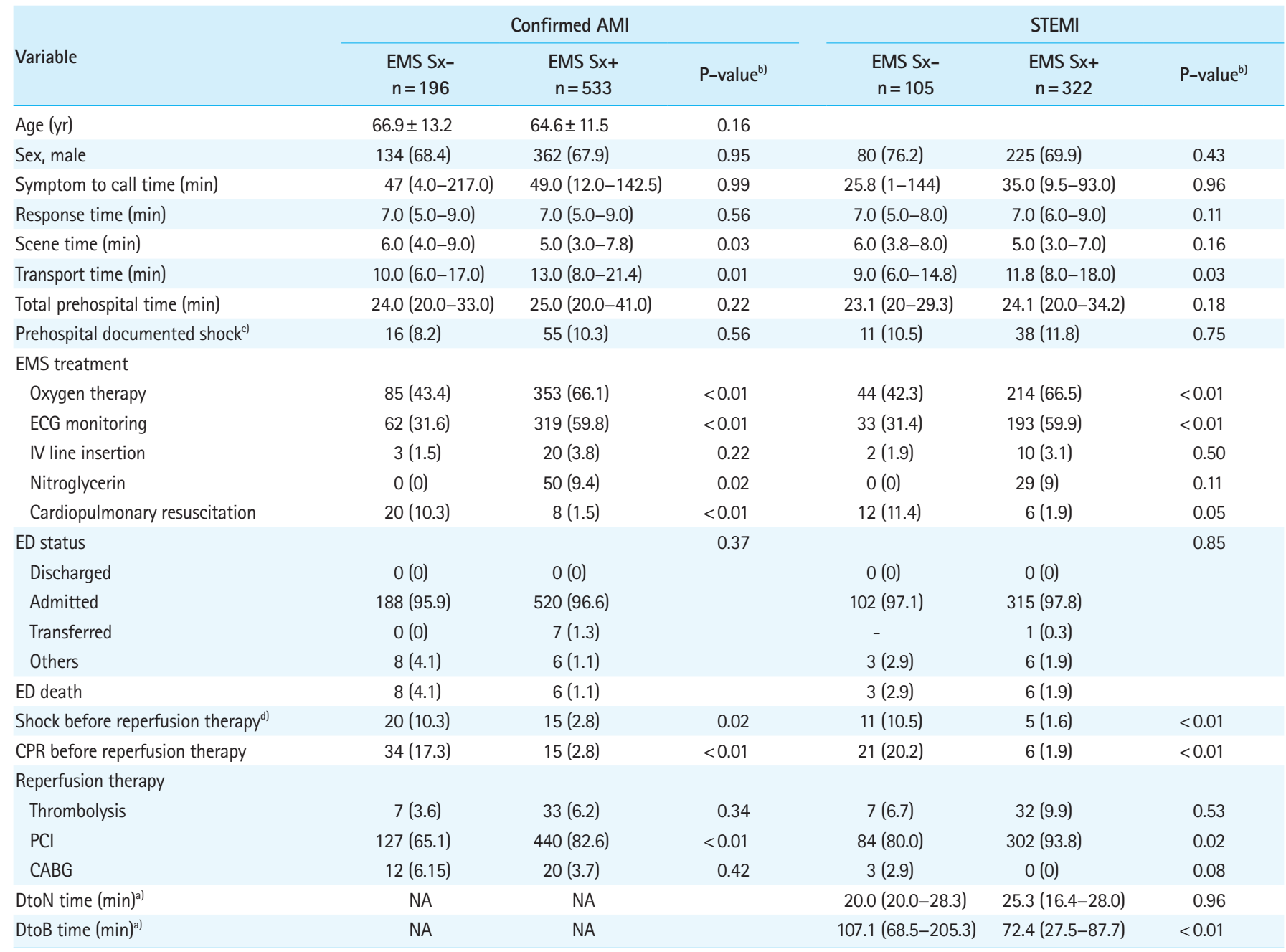

Values are presented as mean \pm standard deviation, number (\%), or median (interquartile range).

ED, emergency department; AMI, acute myocardial infarction; EMS, emergency medical services; STEMI, ST elevation myocardial infarction; EMS Sx-, patients who did not have EMS provider-assessed cardiac symptoms; EMS Sx+, patients who had EMS provider-assessed cardiac symptoms; ECG, electrocardiogram; IV, intravenous; CPR, cardiopulmonary resuscitation; $\mathrm{PCl}$, percutaneous coronary intervention; $\mathrm{CABG}$, coronary artery bypass graft; DtoN time, door-to-needle time in thrombolysis patients; NA, non applicable; DtoB time, door-to-balloon time in primary PCI patients.

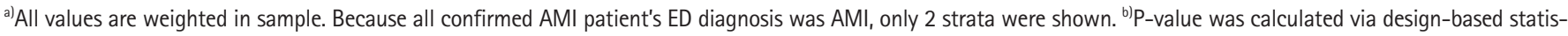

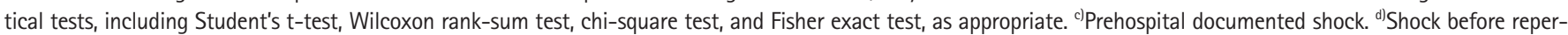
fusion therapy. 


\section{Main outcome}

Table 2 shows the sensitivity, specificity, and predictive values of EMS provider-assessed cardiac symptoms for the diagnosis of AMI. The sensitivity and specificity were $73.3 \%(95 \% \mathrm{Cl}, 70.8$ to $75.7)$ and $85.3 \%(95 \% \mathrm{Cl}, 85.3$ to 85.4$)$, respectively, for any of the EMS-assessed cardiac symptoms, and $65.1 \%(95 \% \mathrm{Cl}, 62.7$ to $67.4)$ and $95.9 \%(95 \% \mathrm{Cl}, 95.9$ to 95.9$)$, respectively, for chest pain. Chest pain was revealed as the most sensitive symptom for diagnosis of AMI, and PPV was also the highest for chest pain (PPV 11.5\%; 95\% Cl, 10.9 to 12.3) (Table 2).

Table 3 shows the prehospital, ED, and hospital characteristics of AMI patients in a weighted sample of EMS-assessed cardiac symptoms. AMI was confirmed in 748 patients $(0.8 \%$ of total patients). Among all confirmed AMI patients, those whose cardiac symptoms were assessed by EMS had shorter scene time and longer transport time and received more oxygen therapy, ECG monitoring, and nitroglycerine administration than patients who had not. The probability of providing CPR in the prehospital or hospital phase was also lower in patients who had EMS-provider assessed cardiac symptoms than in patients who had not. Although prehospital documented shock was more common in patients who had EMS provider-assessed cardiac symptoms than in patients who had not (10.3\% vs. $8.2 \%)$, shock in the hospital phase was less common in patients who had EMS provider-assessed cardiac symptoms than patients who had not (2.8\% vs. $10.3 \%)$. Among STEMI patients, the door-to-balloon time in percutaneous coronary intervention patients was significantly shorter in those who had EMS-assessed cardiac symptoms than in patients who had not (median [interquartile range], 72.4 [27.5 to 87.7] vs. 107.1 [68.5 to 205.3] minutes) (Table 3).

\section{DISCUSSION}

Because the timeliness of treatment is critical in AMI patients, ${ }^{4}$ accurate symptom assessment in the prehospital phase is important. The effectiveness and efficiency of various aspects of prehospital management, including 12-lead ECG readings, oxygen, drugs and fluids, selection of hospital, and prehospital notification, would be improved if symptoms were assessed accurately in the prehospital setting. In this study, we evaluated the diagnostic accuracy of EMS provider-assessed cardiac symptoms for AMI using an EMS database, administrative ED databases, and hospital records. Regardless of individual symptoms, the specificity of EMS-assessed cardiac symptoms was significantly higher than the sensitivity of EMS-assessed cardiac symptoms. This means that these symptoms are more useful at excluding rather than including AMI. For example, if we use chest pain for identifying
$\mathrm{AMI}$, a negative result is reliable at reassuring that a patient does not have AMI (NPV 99.7\%), and chest pain as an indicator of AMI correctly identifies $96 \%$ of patients who do not have AMI (specificity 95.9\%). However, the presence of chest pain is poor at identifying AMI (PPV 11.5\%), and further investigation is needed to diagnose AMI.

We found that EMS provider-assessed cardiac symptoms had very high NPV. However, because only $0.8 \%$ of patients had confirmed AMI, the high NPV of EMS provider-assessed cardiac symptoms was primarily based on the low prevalence of confirmed AMI patients in the EMS database. We also found that the sensitivities of dyspnea, palpitation, and syncope were low. Therefore, the diagnostic value of these symptoms is minimal for identifying AMI.

We found that AMI patients who had EMS provider-assessed cardiac symptoms tend to receive more prehospital management and have low prevalence of shock in the ED phase. Active management in the prehospital setting can be associated with early stabilization of AMI patients. ${ }^{21}$ STEMI patients who had EMS provider-assessed cardiac symptoms had higher prevalence of receiving reperfusion therapy and faster reperfusion time intervals than patients who did not. EMS has been reported to be associated with a wide use of acute reperfusion therapies and fast time intervals in AMI patients. ${ }^{6}$ Even in EMS users, we found that patients who had EMS provider-assessed cardiac symptoms had a higher proportion of those characteristics than patients who did not.

The mechanism of efficient hospital treatment in patients who had EMS-assessed cardiac symptoms was not directly evaluated in our study. Because patients who had EMS-assessed cardiac symptoms had classic symptoms for AMl, the ease of diagnosis can be associated with efficient treatment in the hospital phase. Other EMS provider activities, including prehospital notification and alerts to ED staff when EMS providers hand over patients, can also be associated with those characteristics. Because prehospital 12-lead ECG was not widely used in our setting, the effect of prehospital 12-lead ECG would be minimal in our study.

To our knowledge, this is the first study to evaluate EMS provider-assessed cardiac symptoms in view of the final diagnosis of AMI. Previously, the concordance of symptoms of myocardial infarction between paramedic and hospital records was evaluated in one study, and they found that the concordance of paramedic prehospital patient care documentation of symptoms was excellent for almost all symptoms, including chest pain. ${ }^{22}$ Those findings could suggest that EMS provider-assessed cardiac symptoms could have similar diagnostic accuracy to hospital physician-assessed cardiac symptoms. However, the actual diagnostic accuracy of EMS provider-assessed cardiac symptoms was not evaluat- 
ed. In one study, the diagnostic accuracy of chest pain assessed by an EMS call center was evaluated. ${ }^{23}$ In that study, the overall rate of AMI was $12 \%$ in patients who called EMS for chest discomfort, and that level was similar to the PPV of chest pain in our study.

Compared to previous studies, our study has several strengths. First, rather than include only patients who had chest pain or AMI diagnosis, we included and sampled all patients responded by EMS. Therefore, we could estimate all aspects of diagnostic accuracy, including sensitivity, specificity, and predictive values. If we only included patients who had chest pain and AMI diagnosis, the specificity could not be calculated. Second, because we used disproportionate stratified sampling, we captured a substantial proportion of AMI patients in our sample. Because less than 1\% of the total patients had AMI, a sample of more than 27,300 patients would be needed to capture 273 confirmed AMI patients, which is the same number of confirmed AMI patients in our study. Although we captured a substantial proportion of AMI patients using disproportionate stratified sampling, we found that baseline characteristics were similar between all patients and weighted values of sample patients (Table 1). Third, because other diagnostic tests, including prehospital 12-lead ECG, are seldom used in Korea, the diagnostic accuracy of EMS provider-assessed cardiac symptoms was minimally influenced by other tests.

EMS provider-assessed symptoms are critical for evaluation and management of AMI patients. The diagnostic accuracy of EMS provider-assessed cardiac symptoms can be used as baseline data for policymakers and quality indicators in prehospital AMI management and AMI education programs for EMS providers. Studies about the association between the diagnostic accuracy of EMS provider-assessed cardiac symptoms, outcomes of AMI patients, and the additional diagnostic value of other tests, including prehospital 12-lead ECG, for the evaluation of AMI in patients with EMS provider-assessed cardiac symptoms would be helpful to evaluate the importance of the EMS system and each EMS intervention in the management of AMI more objectively.

Our study has several limitations. First, it was performed at tertiary hospitals in Korea, and the results may not be generalized to other EMS systems. However, the consistency of findings across five years and four hospitals supports our findings, which may be applicable to different ED settings in these regions. Second, the gold standard of AMI was based on hospital medical review in this study, and there may be some errors. However, many studies on AMI use hospital medical review as the gold standard, ${ }^{24,25}$ and because this study was retrospective, there are limited ways to identify real AMI. Third, the effect of EMS provider-assessed cardiac symptoms on the outcomes of AMI patients can be caused by the selection of the designated hospital, but those effects could not be evaluated in our study. Fourth, approximately $24 \%$ of EMS databases were not linked to ED databases, and this could affect our findings. Moreover, because one-to-one matching was planned, duplicated matching pairs and ambiguous matching pairs were excluded in our study. Fifth, the diagnostic accuracy of the combinations of symptoms was not evaluated in this study. In addition, the characteristics of those symptoms, including severity of pain, were not also evaluated in this study.

In conclusion, each of the EMS provider-assessed cardiac symptoms showed moderate sensitivity, high specificity, low PPV, and very high NPV. We also found that among AMI patients, those who had EMS-assessed cardiac symptoms received more aggressive prehospital management and more efficient in-hospital treatment for AMI. Studies about the association of diagnostic accuracy of EMS provider-assessed cardiac symptoms and outcomes and additional diagnostic value of other tests, including prehospital 12-lead ECG, for the evaluation of AMI in patients with EMS provider-assessed cardiac symptoms are needed.

\section{CONFLICT OF INTEREST}

No potential conflict of interest relevant to this article was reported.

\section{ACKNOWLEDGMENTS}

This study was financially supported by the Woochon Cardio-Neuro-Vascular Research Foundation (2014).

\section{REFERENCES}

1. Castelli WP, Garrison RJ, Wilson PW, Abbott RD, Kalousdian S, Kannel WB. Incidence of coronary heart disease and lipoprotein cholesterol levels. The Framingham Study. JAMA 1986; 256:2835-8.

2. Levi F, Chatenoud L, Bertuccio P, Lucchini F, Negri E, La Vecchia C. Mortality from cardiovascular and cerebrovascular diseases in Europe and other areas of the world: an update. Eur J Cardiovasc Prev Rehabil 2009;16:333-50.

3. Murray CJ, Vos T, Lozano R, et al. Disability-adjusted life years (DALYs) for 291 diseases and injuries in 21 regions, 1990-2010: a systematic analysis for the Global Burden of Disease Study 2010. Lancet 2012;380:2197-223.

4. Steg PG, Bonnefoy E, Chabaud S, et al. Impact of time to treatment on mortality after prehospital fibrinolysis or primary angioplasty: data from the CAPTIM randomized clinical trial. 
Circulation 2003;108:2851-6.

5. Ryan TJ, Anderson JL, Antman EM, et al. ACC/AHA guidelines for the management of patients with acute myocardial infarction: executive summary. A report of the American College of Cardiology/American Heart Association Task Force on Practice Guidelines (Committee on Management of Acute Myocardial Infarction). Circulation 1996;94:2341-50.

6. Canto JG, Zalenski RJ, Ornato JP, et al. Use of emergency medical services in acute myocardial infarction and subsequent quality of care: observations from the National Registry of Myocardial Infarction 2. Circulation 2002;106:3018-23.

7. Park $Y H$, Kang GH, Song BG, et al. Factors related to prehospital time delay in acute ST-segment elevation myocardial infarction. J Korean Med Sci 2012;27:864-9.

8. Morrison L, Brooks S, Sawadsky B, McDonald A, Verbeek PR. Prehospital 12-lead electrocardiography impact on acute myocardial infarction treatment times and mortality: a systematic review. Acad Emerg Med 2006;13:84-9.

9. Brainard AH, Raynovich W, Tandberg D, Bedrick EJ. The prehospital 12-lead electrocardiogram's effect on time to initiation of reperfusion therapy: a systematic review and metaanalysis of existing literature. Am J Emerg Med 2005;23:351-6.

10. Cone DC, Lee $\mathrm{CH}$, Van Gelder C. EMS activation of the cardiac catheterization laboratory is associated with process improvements in the care of myocardial infarction patients. Prehosp Emerg Care 2013;17:293-8.

11. Squire BT, Tamayo-Sarver JH, Rashi P, Koenig W, Niemann JT. Effect of prehospital cardiac catheterization lab activation on door-to-balloon time, mortality, and false-positive activation. Prehosp Emerg Care 2014;18:1-8.

12. European Myocardial Infarction Project Group. Prehospital thrombolytic therapy in patients with suspected acute myocardial infarction. N Engl J Med 1993;329:383-9.

13. Morrison $\sqcup$, Verbeek PR, McDonald AC, Sawadsky BV, Cook DJ. Mortality and prehospital thrombolysis for acute myocardial infarction: a meta-analysis. JAMA 2000;283:2686-92.

14. O'Connor RE, Al Ali AS, Brady WJ, et al. Part 9: acute coronary syndromes. 2015 American Heart Association guidelines update for cardiopulmonary resuscitation and emergency cardiovascular care. Circulation 2015;132(18 Suppl 2):S483-500.
15. Bruyninckx R, Aertgeerts B, Bruyninckx P, Buntinx F. Signs and symptoms in diagnosing acute myocardial infarction and acute coronary syndrome: a diagnostic meta-analysis. Br J Gen Pract 2008;58:105-11.

16. Shin SD, Ahn KO, Song KJ, Park CB, Lee EJ. Out-of-hospital airway management and cardiac arrest outcomes: a propensity score matched analysis. Resuscitation 2012;83:313-9.

17. Shin SD, Suh GJ, Ahn KO, Song KJ. Cardiopulmonary resuscitation outcome of out-of-hospital cardiac arrest in low-volume versus high-volume emergency departments: an observational study and propensity score matching analysis. Resuscitation 2011;82:32-9.

18. Katki HA, Li Y, Edelstein DW, Castle PE. Estimating the agreement and diagnostic accuracy of two diagnostic tests when one test is conducted on only a subsample of specimens. Stat Med 2012;31:436-48.

19. Gravitt PE, Paul P, Katki HA, et al. Effectiveness of VIA, Pap, and HPV DNA testing in a cervical cancer screening program in a peri-urban community in Andhra Pradesh, India. PLoS One 2010;5:e13711.

20. Ferreccio $C$, Barriga Ml, Lagos $M$, et al. Screening trial of human papillomavirus for early detection of cervical cancer in Santiago, Chile. Int J Cancer 2013;132:916-23.

21. Mathew TP, Menown IB, McCarty D, Gracey $H_{\text {, Hill }}$, Adgey AA. Impact of pre-hospital care in patients with acute myocardial infarction compared with those first managed in-hospital. Eur Heart J 2003;24:161-71.

22. Coventry LL, Bremner AP, Williams TA, Jacobs IG, Finn J. Symptoms of myocardial infarction: concordance between paramedic and hospital records. Prehosp Emerg Care 2014;18:393-401.

23. Rawshani A, Larsson A, Gelang C, et al. Characteristics and outcome among patients who dial for the EMS due to chest pain. Int J Cardiol 2014;176:859-65.

24. Lambert L, Brown K, Segal E, Brophy J, Rodes-Cabau J, Bogaty P. Association between timeliness of reperfusion therapy and clinical outcomes in ST-elevation myocardial infarction. JAMA 2010;303:2148-55.

25. Yeh RW, Sidney S, Chandra M, Sorel M, Selby JV, Go AS. Population trends in the incidence and outcomes of acute myocardial infarction. N Engl J Med 2010;362:2155-65. 